\title{
Cloud-based Virtual Desktop Environment for Advanced Online Master's Courses
}

\author{
Steffen Moser \\ steffen.moser@uni-ulm.de \\ Fabian Krapp \\ fabian.krapp@uni-ulm.de \\ Stefanie Bärtele \\ stefanie.baertele@uni-ulm.de \\ Kathrin Wunderlich \\ kathrin.wunderlich@uni-ulm. de
}

\author{
Gabriele Gröger \\ gabriele.groeger@uni-ulm.de \\ Frank Slomka \\ frank.slomka@uni-ulm.de \\ Hermann Schumacher \\ hermann.schumachereuni-ulm.de
}

\author{
School of Advanced Professional Studies (SAPS) \\ Ulm University \\ Albert-Einstein-Allee 45 \\ 89081 Ulm, Germany
}

\begin{abstract}
The demand on online courses to achieve higher education skills and knowledge has been rising during the last years. More and more people study academic subjects in parallel of having part-time or even full-time jobs. One of the most important challenges of e-learning solutions is to reproduce the features of a higher education institution's campus as completely as possible to students who attend online courses. While there is a large market of e-learning tools, the majority of them come with a lecturer-oriented and material-based view only. When using single tools, a lot of aspects are not covered, for example the interaction between learners is often not paid enough attention to. In literature it has been shown that the social interaction of students on a classical university or college campus has a very positive effect on learning. To enable social interaction for online students, typically a conglomerate of software tools has to be provided: Classical e-learning tools to distribute content, tools for video conferencing and tools for social networking. While offering just a selection of tools in parallel might be able to cover the requirements formally, it often introduces unnecessary complexity. Several login procedures, different user interfaces and time-consuming ways of organizing data storage can render tools very unattractive. Especially when your typical addressees are roaming students who spend time for learning in alternating environments, there is a high demand for cloud-based virtual desktop solutions, which provide a fast and simple way to start learning or interacting with fellow students, wherever a student stays at the moment. In this work-in-progress paper we present our idea of a cloud-based virtual desktop environment approach while focusing especially on engineering courses and their requirements.
\end{abstract}

Keywords-E-Learning; Cloud; Online; Courses; Engineering; Master; MOOC

\section{INTRODUCTION}

Students in higher education achieve the skills linked to their aspired degree by attending lectures and additional courses like exercises, seminars and projects. Traditionally this takes place in on-campus programs where the students have to attend the courses physically. Nowadays, more and more people explore academics in alternative forms, an example is

The presented work is part of the project Mod:Master (FKZ: 16OH11027) which has been funded by BMBF (German Federal Ministry of Education and Research) since 2011 within the program Offene Hochschulen: Aufstieg durch Bildung.

978-1-4799-5739-2/14/\$31.00 (C)2014 IEEE pursuing a college or university degree while working in a full-time job in parallel. Of course, these alternative forms of studying make great requirements to the institutions of higher education. E-Learning can be understood as a term for the concept of being able to teach and exercise the same things which are typically done in attendance courses by using electronic communication devices and the Internet. For this reason, an online degree program should be at least as powerful or capable as a regular on-campus course of studies. To achieve that, it is necessary to identify the involved communication mechanisms and to find possibilities to provide the functionality online. It further makes sense to consider the student's situation and time schedule of life, work and studying to make appropriate and useful offers.

In this work-in-progress paper we will describe and show our concept for a cloud-based virtual desktop environment for students in higher educations, especially in master's courses. As our institution also offers an online degree program in engineering, we give special attention to the requirements of these courses.

The paper is structured as follows: In the second section we identify the features of a classical campus university. This is necessary to derive the requirements to online degree programs. In the third section we give a short overview about the state-of-the-art e-learning tools. It is followed by a short presentation of the related work. The fifth section is used to present our approach. The paper closes with a summary and conclusion.

\section{FunCtion OF A CAMPUS}

In online programs, the university campus as a central place of a course does not exist. In a typical campus environment, the temporal grid of the semester disposition, lectures, exercises, exams, etc. are present. In a very first approach, it seems logical to provide these mechanisms to online learning students, which is according to Romiszowski necessary to prevent elearning concepts from failing [1]:

- Accessibility of teaching contents. In the most simple form this means to offer lecture presentations, lecture notes, work sheets containing exercises, references to advanced literature and so on in an online way. 
- Interaction with lecturers and tutors. All forms of feedback from the students like contacting the lecturer or tutor in case of questions, submitting exercises for corrections or grading, giving presentations or discussing about a seminar topic must be handled online. A special challenge with no standard solutions are courses which involve working in laboratories which is quite common in natural and engineering sciences.

- Process Organization. Organizational issues must not be neglected: Students are assigned to studying groups and to courses, exams are coordinated between different modules and so on. This has also to be done online.

However, this represents only the organizational and content-based point of view of the education institutes and overlooks the needs of students. Missing are the social aspects or mechanisms that students use frequently but that are not cast in rules or plans and are therefore difficult to grasp, for example:

- Social interaction with peers. A very important aspect of a classical campus is the social interaction of the students with each other. The concept of social networks can be used to overcome the lack of physical interaction in online degree courses, but the technological platform must be adapted to support the students' needs. Also privacy aspects have to be considered. These aspects have been studied by Johnson and Johnson very exhaustively for many years, see for example [2], [3], [4]. Based on the findings of their studies it is evident that the social interaction of studies has a remarkable impact on learning.

- Shared workspace for collaborative work. More and more work in higher educations must be done by students working in groups. This is desired by the instructors as it helps to develop skills in team-work, scientific discussion and representing hypotheses. Students who cannot meet each other personally need electronic platforms for collaborative work. Sharing documents, doing video conferences or showing notes on a virtual scratchpad are only some of the aspects which have to be implemented online.

- Personal workplace. In on-campus educations students typically have two main physical places where they work: At the university and at home where they have fixed work places (e.g. the students' computer lab and the workplace at home). Students who attend online courses do this for various reasons as given in the introduction. Especially people who have a part-time or full-time job in parallel might study in very different places: At home, while commuting from home to work or vice versa or even in some free time slots at work. In an online degree course it is therefore required to support the students with a virtual desktop environment. Ideally, whenever they open their communication device (personal computer, notebook, tablet computer or smart phone) they have to be able to find all of their relevant materials at one place. Good elearning concepts include a content-management solution which is simple to use and is laid out in the point of view of the students.

We derived requirements to online degree programs from the properties of a traditional campus. In the next section, we discuss in how far e-learning tools available at the market can be used to fulfill the requirements.

\section{State of the ART E-LEARning ToOls}

The market of software solutions for e-learning is remarkably large. In [5] Hart maintains a list of the 100 most popular software tools for learning. Two aspects can be observed:

Firstly, most of the tools have very specific focuses, which means that they cover only specific tasks. For example, the content- and instructor-based tools support the distribution of materials, while they don't support the social interaction. For this reason, e-learning solutions at higher education institutions are regularly based on combinations of tools.

Secondly, these combinations often hardly work together. They all come with individual user interfaces, account databases, with individual storages areas and even with different requirements on the students' and lecturers' devices. This does not fit the approach of the central location that a college or university classically offers by its campus. Standard e-learning tools can even make standard procedures quite timeconsuming: When materials offered via a content distribution tool have to be transferred manually to the tool which allows collaborative work, a lot of time will be spent to manage the version history of a document. This unwanted situation is depicted in Fig. 1: While the offered tools satisfy the students' and lecturers' needs, they involve a lot of organizational and usability problems. This leads to technically highly fragmented solutions whose initial commissioning indeed gets the job done, but the people who use that bunch of tools have to deal with an increasing complexity. This isn't only a problem for users themselves, but also for course providers.

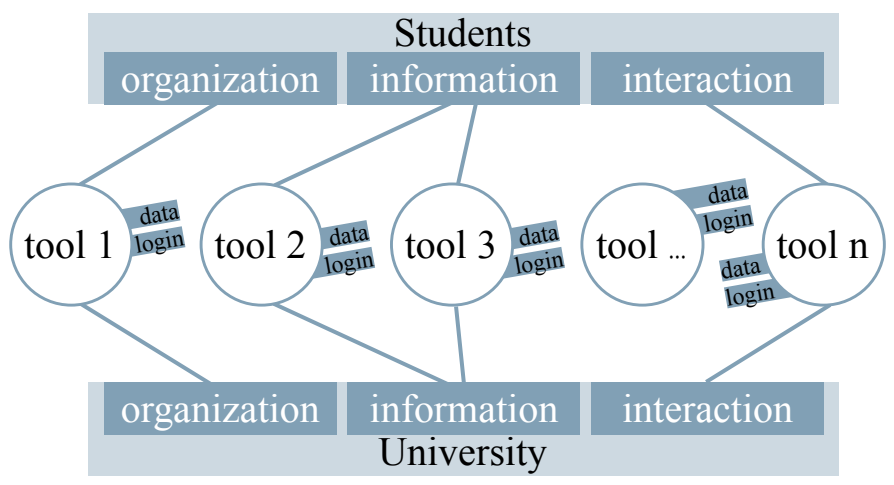

Figure 1. While a plain combination of different e-learning tools offers functionality, the expense for managing all contents and data can easily get too high. The concept of virtual working environments for students are usually neglected.

To overcome this problem, we propose a cloud-based elearning solution.

\section{RELATED WORK}

The lack of holistic e-learning solutions has been often addressed in literature, e.g. by Romiszowski [1] in the year 2004. The needs are further discussed by Watanabe [6]. The possibilities offered by Web 2.0 technologies are presented by Downes [7], [8]. In 2011 Liesebach et al. discussed virtual work-spaces for collaborative e-learning [9]. This is one of the main aspects of our work. [6]

The concept of cloud-based e-learning tools per se is not new. In the last few years also other research groups 
came up with these ideas: Selviandro and Hasibuan propose similar ideas [10]. Additionally, we described a concept to map the interaction of services in the cloud. Oludipe et al. give detailed conceptual ideas about service management and the capabilities of Web 2.0 [11]. This paper is quite interesting because the authors provide courses for natural sciences which are quite similar to engineering courses with respect to their requirements.

\section{Cloud-Based Virtual Desktop EnVironment}

During the last years, cloud computing has been gaining a lot of popularity in information technology. It is a concept where people or corporations source out their compute and data storage jobs to third-party providers. While corporations mostly have the cost-saving aspect in their main focus, people find cloud applications attractive for other reasons: Using cloud services allows people to access their data from wherever they want and with whatever device they have. We show how the concept of cloud computing can be used for e-learning.

To move e-learning to a cloud-based approach, it is at first necessary to identify the actual tasks of the e-learning tools: The base application of those tools used in e-learning environments is to share or make available text, pictures, audio or video. Purposes can be organizational (calendar, management, ...), informational (scripts, instructive media, literature, ...) and interactive (group work, tutorials, chat, ...). So the main reasons to use those tools are basically to exchange of information, make information available and to promote the interaction between participants (text, pictures, audio, video).

The main concept behind a cloud-based approach is to avoid the fragmentation of data and to hide the complexity of the overall system from the user. If not avoided, users have to take the hurdle of the difficult operation of the system even before they access the actual content. Inside the cloud, there are still different tools, but those can be highly inter-related within the cloud. This is shown in Fig. 2. Basically, the user gets one solution that fits all of his needs. Nowadays, it is possible to provide this platform to the user's usual devices: smart phones, tablets computers, notebooks and personal computers.

\section{A. Cloud-Based Tools}

The following e-learning tools are incorporated into our cloud solution:

- OwnCloud [12] is the central application and provides each student or lecturer access to his or her data storage. This storage is used for all services which are described below. Therefore, the student or lecturer has a single point of access to the data stored in the cloud space. This data can be also easily synchronized with one or more local computer systems. OwnCloud's CalDAV-based calendar services are directly used for managing appointments, for example for exams. For each course, a calendar is created which can be also synchronized easily with students' and teachers' mobile devices as it is in a very widespread data format.

- Moodle [13] is used as our main tool to distribute content. Lecture notes, teaching videos and exercises are distributed via Moodle. Moodle is also used to collect submissions of exercises. Short questions regarding the online content can be answered in Moodle via prompts or multiple-choice questions. This helps the student to self-evaluate their learn progress.

- $p h p B B$ [14] is used for discussion boards. While tools like Moodle come with integrated discussion boards, they often lack comfort, i.e. the possibility to cite or to have a proper threading. Specialized discussion board software like phpBB is easier to use. The main reason for setting up a dedicated bulletin board software was the following one: Discussion boards in e-learning tools like Moodle are designed with a strong course-oriented view. Our observation has shown that there is a clear demand of students to discuss not only related to a specific instance of a course, but also more globally.

- Big Blue Button [15] is an open-source tool for browserbased video and audio conferencing. Especially in engineering courses, it is necessary to provide online office hours where students have the possibility to ask the tutor or the lecturer to demonstrate selected, typically mathematicsrelated exercises. Therefore, we linked Big Blue Button to an interactive electronic whiteboard which can be included into the video conference. Due to the cloud approach, the web conferences between students and teachers can be recorded and are typically be published automatically as course materials. Big Blue Button also satisfies a second requirement: It is a platform for students to meet in conference rooms with each other. This is, for example, used for discussing exercises and preparing seminar papers together.

- Remote Tool Service is a tool which is based on the open source software Guacamole [16]. It allows our students to connect to virtual Linux or Windows desktops using the web browser on their devices and is depicted in Fig. 3. As the solution is fully HTML 5-based, this works with all modern communication devices. The reason for offering this service is that in engineering courses, it is quite common to work with course-specific software tools. This can be numerical solvers, advanced computer algebra systems, modeling and simulation frameworks and so on. As it is typically quite time-consuming to install and configure these large tools on students' individual platforms, offering the students only a download of a campus-wide licensed version of such tools does not encourage them to actually download, install and configure them. The remote tool service gives students the ability to have their fullyequipped lab computer only a few clicks away. Running sessions can be handed over from one device to another without having to exit the remotely running programs. All data which are stored by a tool executed in the remote tool service will be part of the logged-in student's personal cloud storage and can be easily accessed from everywhere via OwnCloud as mentioned above.

\section{B. Technical Realization}

One of the most important technical parts of the cloud solution is a common account and authentication source. For all cloud-based applications a common user database is needed. In our case this is accomplished by using an OpenLDAP [17] directory. However, this is by far not enough. OpenLDAP itself does not come with a user-friendly possibility for password self-service. Especially when working with large user bases it 


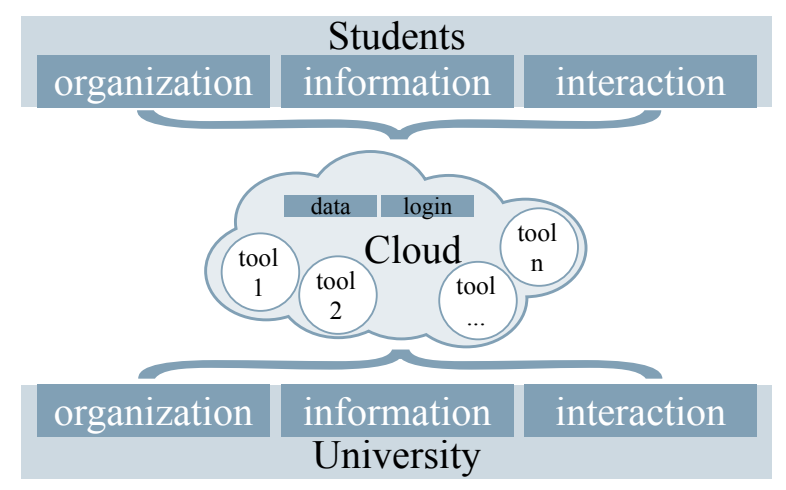

Figure 2. In the cloud-based approach, the e-learning tools needed are administratively grouped to a cloud computing concept. This allows students and teachers to connect to the system with a single entry point which enables, for example, the concept of a fully integrated data and document storage management - also for roaming users.

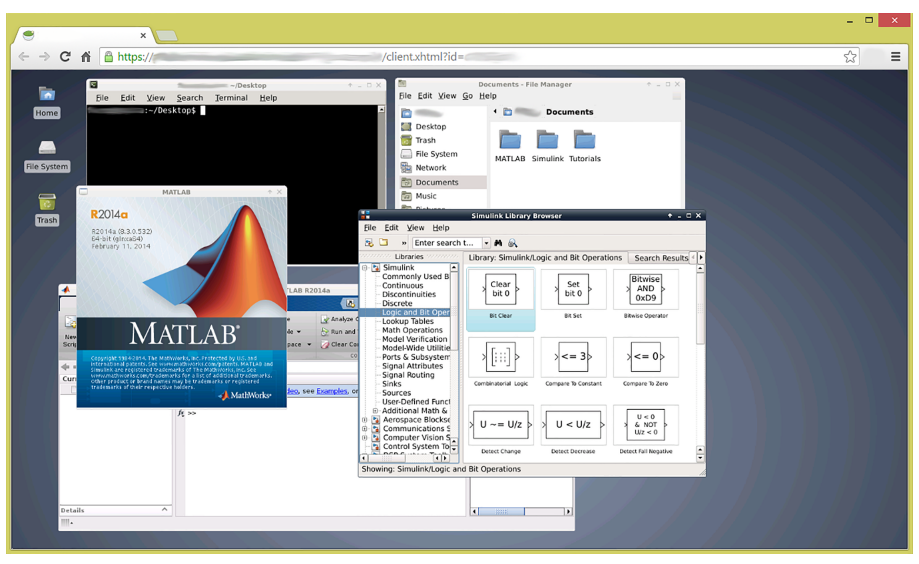

Figure 3. A remote tool service session allows roaming students to work with laboratory tools used in engineering exercises. These tools typically introduce a large time and effort for installation and configuration on local computers. In our approach these tools can be used fully browser-based without the need of any installation works. The file system is linked to the common data and document storage in the cloud.

is eminent that the users are able to handle password problems by themselves. For this reason, we extended OpenLDAP by the password self-service $p w m$ [18]. Regarding the cloudbased application's side, OpenLDAP allows a common user database, but nevertheless, each service will still do its own authentication procedure. This leads to multiple user name and password requests which are actually not necessary. To avoid this, Apereo JASIG CAS [19] as a Central Authentication System has been installed on-top of OpenLDAP. CAS allows web applications to form a trust relationship between each other. This results in a real single-sign-on solution which improves the usability a lot: The different components of the elearning environment are perceived as a rather holistic solution. Besides storing the user information, it is necessary to handle the data. Caused by working with high-definition teaching videos, we have to deal with large amounts of data. For this purpose, a high-performance ZFS file system on a Oracle Solaris 11 [20] server infrastructure is used. The concept of Solaris zones protects services from each other without much overhead. Reliability of the cloud system is reached by using redundant disk systems.

\section{CONCLUSION AND OUTLOOK}

The work presented in this paper is currently still under development. As the next step we plan a round-up of the graphical user interfaces used in the tools. We also look forward to do a evaluation with our online students to find out more about the acceptance and the actual usage of the tools. We especially need to know about services that our students still miss, but we are also interested in knowing if there are functions which do have lower priorities than expected.

\section{REFERENCES}

[1] A. J. Romiszowski, "How's the E-learning Baby? Factors Leading to Success of Failure of an Educational Technology Innovation," Educational Technology, vol. 44, no. 1, pp. 5-27, 2004.

[2] D. W. Johnson and R. T. Johnson, "Cooperation and Competition: Theory and Research," 1989.

[3] — "Cooperative Learning and Research," Cooperative learning theory and research, pp. 23-37, 1990.

[4] - "An Educational Psychology Success Story: Social Interdependence Theory and Cooperative Learning," Educational Researcher, vol. 38, no. 5, pp. 365-379, 2009. [Online]. Available: http://edr.sagepub.com/content/38/5/365.abstract

[5] J. Hart. Top 100 Tools for Learning. Last accessed: 2014-09-18. [Online]. Available: http://c4lpt.co.uk/top100tools/

[6] K. Watanabe, "A study on the needs for e-Learning: Through the analysis of national survey and case studies," Progress in Informatics, vol. 2, pp. 77-86, 2005.

[7] S. Downes, "E-learning 2.0," vol. 2005, no. 10. New York, NY, USA: ACM, Oct. 2005, pp. 1-. [Online]. Available: http: //doi.acm.org/10.1145/1104966.1104968

[8] _ - "Learning networks in practice," D. Ley, Ed., vol. 2. London: BECTA, 2007, p. 19-27.

[9] K. Liesebach, E. Franz, A.-K. Stange, A. Juschka, K. BorceaPfitzmann, A. Böttcher, and H. Wahrig, "Collaborative E-Learning," in Digital Privacy, ser. Lecture Notes in Computer Science, J. Camenisch, R. Leenes, and D. Sommer, Eds. Springer Berlin Heidelberg, 2011, vol. 6545, pp. 657-677. [Online]. Available: http://dx.doi.org/10.1007/978-3-642-19050-6_24

[10] N. Selviandro and Z. Hasibuan, "Cloud-Based E-Learning: A Proposed Model and Benefits by Using E-Learning Based on Cloud Computing for Educational Institution," in Information and Communication Technology, ser. Lecture Notes in Computer Science, K. Mustofa, E. Neuhold, A. Tjoa, E. Weippl, and I. You, Eds. Springer Berlin Heidelberg, 2013, vol. 7804, pp. 192-201. [Online]. Available: http://dx.doi.org/10.1007/978-3-642-36818-9_20

[11] O. Oludipe, O. Fatoki, N. Yekini, and A. E.E., "Cloud-based E-Learning Platform: From the Perspective of "Structure" and "Interaction"," International Journal of Innovation and Research in Educational Sciences, vol. $1,2014$.

[12] ownCloud.org. Last accessed: 2014-09-18. [Online]. Available: http: //owncloud.org/

[13] Moodle - Open-source learning platform - Moodle.org. Last accessed: 2014-09-18. [Online]. Available: http://moodle.org/

[14] phpBB. Last accessed: 2014-09-18. [Online]. Available: https: //www.phpbb.com/

[15] BigBlueButton. Last accessed: 2014-09-18. [Online]. Available: http://bigbluebutton.org/

[16] Guacamole - HTML5 Clientless Remote Desktop. Last accessed: 2014-09-18. [Online]. Available: http:/guac-dev.org/

[17] OpenLDAP. Last accessed: 2014-09-18. [Online]. Available: http: //www.openldap.org/

[18] pwm - Open Source Password Self Service for LDAP directories. Last accessed: 2014-09-18. [Online]. Available: https://code.google.com/p/ pwm/

[19] CAS - Apereo Foundation. Last accessed: 2014-09-18. [Online]. Available: https://www.apereo.org/cas

[20] Oracle Solaris 11. Last accessed: 2014-09-18. [Online]. Available: http://www.oracle.com/solaris 11 